Commun. Fac. Sci. Univ. Ank. Ser. A1 Math. Stat.

Volume 69, Number 1, Pages 158-171(2020)

DOI: $10.31801 /$ cfsuasmas. 532747

ISSN 1303-5991 E-ISSN 2618-6470

http://communications.science.ankara.edu.tr/index.php?series=A1

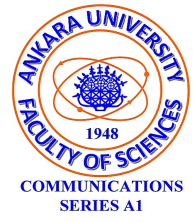

\title{
ANALYSIS OF THE RAYLEIGH WAVE FIELD DUE TO A TANGENTIAL LOAD APPLIED ON THE SURFACE OF A COATED ELASTIC HALF-SPACE
}

\author{
ONUR ŞAHIN
}

\begin{abstract}
The paper deals with 3D dynamic response of a coated elastic half space subject to in-plane surface loading. The problem is formulated by a pair of elliptic equations over the interior and a two dimensional singularly perturbed hyperbolic equation expressed in terms of shear wave potentials along the interface. As an example, a point force acting one of the in-plane axis is considered and the integral solution of the normal displacement along the interface is derived through the use of the relation between the wave potentials.
\end{abstract}

\section{INTRODUCTION}

Propagation of surface waves has been the focus of intensive research since its introduction by the monumental work of Rayleigh [1. Rayleigh waves, therefore, have been extensively studied by scientists and engineers due to their applicability to acoustic, seismology, electromagnetism, among others. One of the most important contribution to the Rayleigh wave was made by Friedlander who presented the Rayleigh wave field for an elastic half plane in terms of arbitrary plane harmonic functions [2]. In a later publication, Chadwick extended Friedlander's analysis and expressed the Rayleigh wave field in terms of a single harmonic function via a relation between the wave potentials at the surface of the elastic half-plane [3]. This relationship was, then, extended to three dimensions in [4.

The significance of the surface waves on an elastic half-plane or half-space motivates an alternative analysis under more general assumptions, which may help to extract the Rayleigh wave contribution. Therefore, recent studies have generally focused on employing approximate models to derive the Rayleigh wave contribution which is often hidden in the problem formulation, see, e.g., [5]-8]. In [8, an explicit model for the Rayleigh and Bleustein-Gulyaev surface waves was presented. The derivations were based on perturbing in slow time the self-similar solution for

Received by the editors: February 26, 2019; Accepted: September 30, 2019.

2010 Mathematics Subject Classification. Primary 05C38, 74J15; Secondary 74G10, 74B05.

Key words and phrases. Rayleigh wave, thin coating, asymptotic model, tangential load.

Communications Faculty of Sciences University of Ankara-Series (C2020 Ankara University 


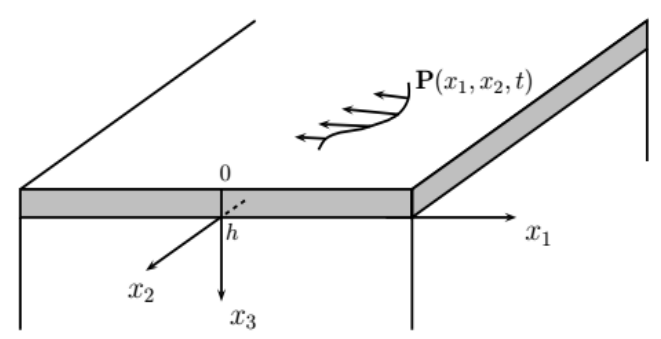

Figure 1. Tangential loading on the surface of coated half-space

homogeneous surface waves given in $[2$ and 3 . Thus, the developed models for the surface waves consisted of hyperbolic equation on the surface with two elliptic equations in the interior domain. The formulation in 8 was later generalized to the three dimensional linear, isotropic, coated elastic half-space taking into account the effect of a thin coating [9]. The hyperbolic-elliptic model for surface wave on an orthorhombic half space was presented in [10. A surface wave of arbitrary profile in anisotropic half-space was constructed by means of the Stroh formalism in [11. We also mention [12] which considered surface waves in a coated half-space with a clamped surface because of the applicability of the hyperbolic-elliptic model for surface wave in high frequency domain. Analysis of the Rayleigh field in a three dimensional elastic half space subject to in-plane surface loading was given in 13]. In addition we cite [14 summarizing the asymptotic model for Rayleigh and Rayleigh type waves and [15] which includes a recent composite model joining both low-frequency and high-frequency models. Along with the latest advancement of technology, moving load problems also find various modern industrial applications in modern engineering, see [16]-19]. The developed hyperbolic elliptic models have also been utilized for investigation of the near-resonant regimes of moving loads on elastic and coated elastic half-spaces, see e.g [20]-24].

The organization of the paper is described as follows. Section 2 contains the statement of the problem, presenting the governing equations together with the boundary conditions. In Section 3 an asymptotic model for the Rayleigh wave field in the case of an elastic half-space coated with a thin layer is developed. An illustrative example for the derived model is given in Section 4. In the last section numerical computations based on the derived approximate formulae are presented.

\section{Statement of the PRoblem}

Consider a 3D homogeneous isotropic elastic half-space coated by a thin layer of constant thickness $h$, loaded with a tangential force of amplitude $P$, see Figure 1 . The equations of motion in 3D elasticity are written as (see, e.g. [25])

$$
(\lambda+\mu) \operatorname{grad} \operatorname{div} \mathbf{u}+\mu \Delta \mathbf{u}=\rho \mathbf{u}_{t t},
$$


where $\mathbf{u}=\left(u_{1}, u_{2}, u_{3}\right)$ is the displacement vector, $\rho$ is the volume density and $\Delta$ is the 3D Laplace operator.

The constitutive relations for a linear isotropic elastic solid are given by

$$
\sigma_{i j}=\lambda \delta_{i j} \operatorname{div} \mathbf{u}+\mu\left(\frac{\partial u_{i}}{\partial x_{j}}+\frac{\partial u_{j}}{\partial x_{i}}\right), \quad i=1,2,3,
$$

where $\sigma_{i j}$ are the components of the Cauchy stress tensor, $\lambda$ and $\mu$ are the Lamé constants and $\delta_{i j}$ is the Kronecker delta.

The boundary conditions at the surface $x_{3}=0$ of the coating are specified as

$$
\left(\sigma_{13}, \sigma_{23}, \sigma_{33}\right)=-\mathbf{P} .
$$

The tangential load may be decomposed through the Helmholtz theorem [25] as

$$
\mathbf{P}\left(x_{1}, x_{2}, t\right)=\left(\boldsymbol{\nabla} P_{0}+\boldsymbol{\nabla} \times \tilde{\mathbf{P}}\right)=\left(P^{(1)}, P^{(2)}, 0\right),
$$

where

$$
\tilde{\mathbf{P}}=\left(0,0, P_{1}\right) \text { and }\left(P^{(1)}, P^{(2)}\right)=\left(\frac{\partial P_{0}}{\partial x_{1}}+\frac{\partial P_{1}}{\partial x_{2}}, \frac{\partial P_{0}}{\partial x_{2}}-\frac{\partial P_{1}}{\partial x_{1}}\right) .
$$

All of the equations above describe the substrate $x_{3} \geqslant h$. In the case of coating, $0 \leq x_{3} \leq h$, subscript " 0 " is used for the material parameters, e.g. $\rho_{0}, \lambda_{0}, \mu_{0}$ etc.

Our first aim is to state the boundary conditions at the interface $x_{3}=h$. To this end, taking into consideration the effective boundary conditions presented in 9], the boundary conditions at the surface $x_{3}=0$ may be carried on the surface of the substrate. As a result, the boundary conditions at $x_{3}=h$ can be written as

$$
\begin{aligned}
\sigma_{i 3}=\mu\left(\frac{\partial u_{i}}{\partial x_{3}}+\frac{\partial u_{3}}{\partial x_{i}}\right) & =\rho_{0} h\left\{\frac{\partial^{2} u_{i}}{\partial t^{2}}-c_{20}^{2}\left(\frac{\partial^{2} u_{3}}{\partial x_{j}^{2}}+4\left(1-\kappa_{0}^{-2}\right) \frac{\partial^{2} u_{i}}{\partial x_{i}^{2}}\right.\right. \\
& \left.\left.+\left(3-4 \kappa_{0}^{-2}\right) \frac{\partial^{2} u_{j}}{\partial x_{i} \partial x_{j}}\right)\right\}-P^{(i)},
\end{aligned}
$$

and

$$
\sigma_{33}=\lambda\left(\frac{\partial u_{i}}{\partial x_{i}}+\frac{\partial u_{j}}{\partial x_{j}}\right)+(\lambda+2 \mu) \frac{\partial u_{3}}{\partial x_{3}}=\rho_{0} h \frac{\partial^{2} u_{3}}{\partial t^{2}}, \quad i \neq j=1,2
$$

where $c_{10}, c_{20}$ are the longitudinal and transverse wave speeds, $\rho_{0}$ is the density of the coating and $\kappa_{0}=c_{10} / c_{20}$.

\section{Asymptotic Model}

In this section an asymptotic model is established for the substrate governed by equation (1) and subject to the effective boundary conditions (6)-(7) at the surface $x_{3}=h$. First, on applying the Radon transform to the equations of motion (1), see [26], the problem is reduced to a two-dimensional one and, then, the explicit model, derived for an elastic half-plane in [8, is applied to the reduced two-dimensional boundary value problem. Thus, an elliptic-hyperbolic model may be developed for the considered problem. 
Let us apply the Radon transform to eqs. (17, (6) and (7), resulting, respectively, in

$$
\begin{gathered}
{\left[(\lambda+\mu) \cos ^{2} \alpha+\mu\right] \frac{\partial^{2} u_{1}^{(\alpha)}}{\partial \chi^{2}}+\mu \frac{\partial^{2} u_{1}^{(\alpha)}}{\partial x_{3}^{2}}} \\
+(\lambda+\mu) \cos \alpha\left(\sin \alpha \frac{\partial^{2} u_{2}^{(\alpha)}}{\partial \chi^{2}}+\frac{\partial^{2} u_{3}^{(\alpha)}}{\partial \chi \partial x_{3}}\right)=\rho \frac{\partial^{2} u_{1}^{(\alpha)}}{\partial t^{2}} \\
{\left[(\lambda+\mu) \sin ^{2} \alpha+\mu\right] \frac{\partial^{2} u_{2}^{(\alpha)}}{\partial \chi^{2}}+\mu \frac{\partial^{2} u_{2}^{(\alpha)}}{\partial x_{3}^{2}},} \\
+(\lambda+\mu) \sin \alpha\left(\cos \alpha \frac{\partial^{2} u_{1}^{(\alpha)}}{\partial \chi^{2}}+\frac{\partial^{2} u_{3}^{(\alpha)}}{\partial \chi \partial x_{3}}\right)=\rho \frac{\partial^{2} u_{2}^{(\alpha)}}{\partial t^{2}}, \\
(\lambda+\mu)\left(\cos \alpha \frac{\partial^{2} u_{1}^{(\alpha)}}{\partial \chi \partial x_{3}}+\sin \alpha \frac{\partial^{2} u_{2}^{(\alpha)}}{\partial \chi \partial x_{3}}\right)+\mu \frac{\partial^{2} u_{3}^{(\alpha)}}{\partial \chi^{2}}, \\
+(\lambda+2 \mu) \frac{\partial^{2} u_{3}^{(\alpha)}}{\partial x_{3}^{2}}=\rho \frac{\partial^{2} u_{3}^{(\alpha)}}{\partial t^{2}}
\end{gathered}
$$

and

$$
\begin{aligned}
\sigma_{13}^{(\alpha)}=\mu & \left(\frac{\partial u_{1}^{(\alpha)}}{\partial x_{3}}+\cos \alpha \frac{\partial u_{3}^{(\alpha)}}{\partial \chi}\right)=\rho_{0} h\left[\frac{\partial^{2} u_{1}^{(\alpha)}}{\partial t^{2}}-c_{20}^{2}\left(\sin ^{2} \alpha \frac{\partial^{2} u_{1}^{(\alpha)}}{\partial \chi^{2}}\right.\right. \\
& \left.\left.+4\left(1-\kappa_{0}^{-2}\right) \cos ^{2} \alpha \frac{\partial^{2} u_{1}^{(\alpha)}}{\partial \chi^{2}}+\left(3-4 \kappa_{0}^{-2}\right) \sin \alpha \cos \alpha \frac{\partial^{2} u_{2}^{(\alpha)}}{\partial \chi^{2}}\right)\right] \\
& -\left(\cos \alpha \frac{\partial P_{0}^{(\alpha)}}{\partial \chi}+\sin \alpha \frac{\partial P_{1}^{(\alpha)}}{\partial \chi}\right), \\
\sigma_{23}^{(\alpha)}=\mu & \left(\frac{\partial u_{2}^{(\alpha)}}{\partial x_{3}}+\sin \alpha \frac{\partial u_{3}^{(\alpha)}}{\partial \chi}\right)=\rho_{0} h\left[\frac{\partial^{2} u_{2}^{(\alpha)}}{\partial t^{2}}-c_{20}^{2}\left(\cos ^{2} \alpha \frac{\partial^{2} u_{2}^{(\alpha)}}{\partial \chi^{2}}\right.\right. \\
+ & \left.\left.4\left(1-\kappa_{0}^{-2}\right) \sin ^{2} \alpha \frac{\partial^{2} u_{2}^{(\alpha)}}{\partial \chi^{2}}+\left(3-4 \kappa_{0}^{-2}\right) \sin \alpha \cos \alpha \frac{\partial^{2} u_{1}^{(\alpha)}}{\partial \chi^{2}}\right)\right] \\
& -\left(\sin \alpha \frac{\partial P_{0}^{(\alpha)}}{\partial \chi}-\cos \alpha \frac{\partial P_{1}^{(\alpha)}}{\partial \chi}\right), \\
& \left(\cos \alpha \frac{\partial u_{1}^{(\alpha)}}{\partial \chi}+\sin \alpha \frac{\partial u_{2}^{(\alpha)}}{\partial \chi}\right)+(\lambda+2 \mu) \frac{\partial u_{3}^{(\alpha)}}{\partial x_{3}}=\rho_{0} h \frac{\partial^{2} u_{3}^{(\alpha)}}{\partial t^{2}} .
\end{aligned}
$$


Here, the Radon transform is defined as

$$
u_{k}^{(\alpha)}\left(\chi, \alpha, x_{3}, t\right)=\int_{-\infty}^{\infty} u_{k}\left(\chi \cos \alpha-\zeta \sin \alpha, \chi \sin \alpha+\zeta \cos \alpha, x_{3}, t\right) d \zeta
$$

where

$$
\chi=x_{1} \cos \alpha+x_{2} \sin \alpha, \quad \zeta=-x_{1} \sin \alpha+x_{2} \cos \alpha
$$

with the angle $\alpha$ varying on the interval $0 \leq \alpha \leq 2 \pi$, see Figure 2 The original

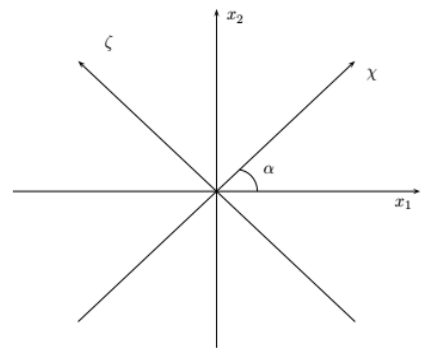

Figure 2. Rotation of Cartesian frame

displacements may be written in terms of the transformed displacements as

$$
u_{1}=u_{\chi}^{\alpha} \cos (\alpha)-u_{\zeta}^{\alpha} \sin (\alpha), \quad u_{2}=u_{\chi}^{\alpha} \sin (\alpha)+u_{\zeta}^{\alpha} \cos (\alpha) .
$$

Assuming that the surface wave field is not distributed by anti-plane motion, it can be emphasized that $u_{\zeta}^{\alpha}=0$, see [9. Substituting eq. (11) into eq. (8) and taking into account the assumption above, eq. (8) takes the following plane problem form:

$$
\begin{aligned}
& (\lambda+2 \mu) \frac{\partial^{2} u_{\chi}^{(\alpha)}}{\partial \chi^{2}}+\mu \frac{\partial^{2} u_{\chi}^{(\alpha)}}{\partial x_{3}^{2}}+(\lambda+\mu) \frac{\partial^{2} u_{3}^{(\alpha)}}{\partial \chi \partial x_{3}}=\rho \frac{\partial^{2} u_{\chi}^{(\alpha)}}{\partial t^{2}}, \\
& (\lambda+\mu) \frac{\partial^{2} u_{\chi}^{(\alpha)}}{\partial \chi \partial x_{3}}+\mu \frac{\partial^{2} u_{3}^{(\alpha)}}{\partial \chi^{2}}+(\lambda+2 \mu) \frac{\partial^{2} u_{3}^{(\alpha)}}{\partial x_{3}^{2}}=\rho \frac{\partial^{2} u_{\chi}^{(\alpha)}}{\partial t^{2}},
\end{aligned}
$$

with the boundary conditions

$$
\begin{aligned}
\sigma_{\chi 3}^{(\alpha)} & =\mu\left(\frac{\partial u_{\chi}^{(\alpha)}}{\partial x_{3}}+\frac{\partial u_{3}^{(\alpha)}}{\partial \chi}\right)=\mu_{0} h\left[c_{20}^{-2} \frac{\partial^{2} u_{\chi}^{(\alpha)}}{\partial t^{2}}-4\left(1-\kappa_{0}^{-2}\right) \frac{\partial^{2} u_{\chi}^{(\alpha)}}{\partial \chi^{2}}\right]-\frac{\partial P_{0}^{(\alpha)}}{\partial \chi}, \\
\sigma_{33}^{(\alpha)} & =\lambda \frac{\partial u_{\chi}^{(\alpha)}}{\partial \chi}+(\lambda+2 \mu) \frac{\partial u_{3}^{(\alpha)}}{\partial x_{3}}=\rho_{0} h \frac{\partial^{2} u_{3}^{(\alpha)}}{\partial t^{2}} .
\end{aligned}
$$

It is well known that the displacement vector $\mathbf{u}$ may expressed through the sum of gradient of scaler potential $\phi$ and the curl of a vector potential $\psi$, that is

$$
\mathbf{u}=\boldsymbol{\nabla} \phi+\nabla \times \boldsymbol{\psi},
$$


where $\boldsymbol{\psi}=\left(-\psi_{2}, \psi_{1}, 0\right)$, see [25] and 9]. Thus, the transformed displacement components can be expressed in terms of the transformed wave potentials as

$$
u_{\chi}^{(\alpha)}=\frac{\partial \phi^{(\alpha)}}{\partial \chi}-\frac{\partial \psi^{(\alpha)}}{\partial x_{3}} \quad \text { and } \quad u_{3}^{(\alpha)}=\frac{\partial \phi^{(\alpha)}}{\partial x_{3}}+\frac{\partial \psi^{(\alpha)}}{\partial \chi} .
$$

On inserting the transformed potentials into displacement and boundary equations (12) and 13 we obtain

$$
\begin{aligned}
& \frac{\partial^{2} \phi^{(\alpha)}}{\partial \chi^{2}}+\frac{\partial^{2} \phi^{(\alpha)}}{\partial x_{3}^{2}}-\frac{1}{c_{1}^{2}} \frac{\partial^{2} \phi^{(\alpha)}}{\partial t^{2}}=0, \\
& \frac{\partial^{2} \psi^{(\alpha)}}{\partial \chi^{2}}+\frac{\partial^{2} \psi^{(\alpha)}}{\partial x_{3}^{2}}-\frac{1}{c_{2}^{2}} \frac{\partial^{2} \psi^{(\alpha)}}{\partial t^{2}}=0,
\end{aligned}
$$

and

$$
\begin{aligned}
& \mu\left[2 \frac{\partial^{2} \phi^{(\alpha)}}{\partial \chi \partial x_{3}}+\frac{\partial^{2} \psi^{(\alpha)}}{\partial \chi^{2}}-\frac{\partial^{2} \psi^{(\alpha)}}{\partial x_{3}^{2}}\right]= \mu_{0} h\left[c_{20}^{-2}\left(\frac{\partial^{3} \phi^{(\alpha)}}{\partial \chi \partial t^{2}}-\frac{\partial^{3} \psi^{(\alpha)}}{\partial x_{3} \partial t^{2}}\right)\right. \\
&\left.-4\left(1-\kappa_{0}^{-2}\right)\left(\frac{\partial^{3} \phi^{(\alpha)}}{\partial \chi^{3}}-\frac{\partial^{3} \psi^{(\alpha)}}{\partial x_{3} \partial \chi^{2}}\right)\right]-\frac{\partial P_{0}^{(\alpha)}}{\partial \chi} \\
& \mu\left[\left(\kappa^{2}-2\right) \frac{\partial^{2} \phi^{(\alpha)}}{\partial \chi^{2}}+\kappa^{2} \frac{\partial^{2} \phi^{(\alpha)}}{\partial x_{3}^{2}}+2 \frac{\partial^{2} \psi^{(\alpha)}}{\partial \chi \partial x_{3}}\right]=\mu_{0} h c_{20}^{-2}\left(\frac{\partial^{3} \phi^{(\alpha)}}{\partial x_{3} \partial t^{2}}+\frac{\partial^{3} \psi^{(\alpha)}}{\partial \chi \partial t^{2}}\right) .
\end{aligned}
$$

where $c_{1}$ and $c_{2}$ are the longitudinal and shear wave speeds and $\kappa=c_{1} / c_{2}$. It can easily be seen from the boundary equations that the surface wave is only induced by gradient part of the applied load $P_{0}$.

The considered three dimensional problem of elasticity, thus, is reduced to a two dimensional plane problem with the help of the Radon transform. We can now employ the explicit model dealing with the wave propagation along the surface of the two dimensional elastic half-plane with the Rayleigh wave speed, see 8 . Following the same asymptotic methodology performed in [8] and [9], the wave equations (16) are cast into a pair of elliptic equations in the interior of the half plane, given by

$$
\begin{aligned}
& \frac{\partial^{2} \phi^{(\alpha)}}{\partial x_{3}^{2}}+k_{1}^{2} \frac{\partial^{2} \phi^{(\alpha)}}{\partial \chi^{2}}=0 \\
& \frac{\partial^{2} \psi^{(\alpha)}}{\partial x_{3}^{2}}+k_{2}^{2} \frac{\partial^{2} \psi^{(\alpha)}}{\partial \chi^{2}}=0 .
\end{aligned}
$$


Similarly, the boundary conditions 17 give a partial differential equation along the surface $x_{3}=h$

$$
\frac{\partial^{2} \psi^{(\alpha)}}{\partial \chi^{2}}-\frac{1}{c_{R}^{2}} \frac{\partial^{2} \psi^{(\alpha)}}{\partial t^{2}}+\frac{b h}{k_{2}} \frac{\partial^{3} \psi^{(\alpha)}}{\partial \chi^{2} \partial x_{3}}=\frac{\left(1+k_{2}^{2}\right)}{2 \mu B} \frac{\partial P_{0}^{(\alpha)}}{\partial \chi},
$$

with the relation between the wave potentials on the surface expressed through

$$
\frac{\partial \psi^{(\alpha)}}{\partial \chi}=-\frac{2}{1+k_{2}^{2}} \frac{\partial \phi^{(\alpha)}}{\partial x_{3}}, \quad \text { or } \quad \frac{\partial \phi^{(\alpha)}}{\partial \chi}=\frac{2}{1+k_{2}^{2}} \frac{\partial \psi^{(\alpha)}}{\partial x_{3}},
$$

where $k_{i}^{2}=1-c_{R}^{2} / c_{i}^{2} ; i=1,2, m=\mu_{0} / \mu$ and

$$
\begin{aligned}
& B=\left(1-k_{1}^{2}\right) \frac{k_{2}}{k_{1}}+\left(1-k_{2}^{2}\right) \frac{k_{1}}{k_{2}}-\left(1-k_{2}^{4}\right), \\
& b=\frac{m}{2 B}\left(1-k_{2}^{2}\right)\left[\left(1-k_{20}^{2}\right)\left(k_{1}+k_{2}\right)-4 k_{2}\left(1-\kappa_{0}^{-2}\right)\right] .
\end{aligned}
$$

By taking the inverse Radon transform of eqns. (18)-20 we arrive at the asymptotic formulation given by two elliptic equations in the interior

$$
\frac{\partial^{2} \phi}{\partial x_{3}^{2}}+k_{1}^{2} \Delta_{2} \phi=0, \quad \frac{\partial^{2} \psi_{i}}{\partial x_{3}^{2}}+k_{1}^{2} \Delta_{2} \psi_{i}=0, \quad i=1,2,
$$

with the surface equation along the plane $x_{3}=h$

$$
\Delta_{2} \psi_{i}-\frac{1}{c_{R}^{2}} \frac{\partial^{2} \psi_{i}}{\partial t^{2}}-b h \sqrt{-\Delta_{2}} \Delta_{2} \psi_{i}=\frac{\left(1+k_{2}^{2}\right)}{2 \mu B} \frac{\partial P_{0}}{\partial x_{i}}, \quad i=1,2,
$$

and the relations between the potentials

$$
\frac{\partial \phi}{\partial x_{i}}=\frac{2}{1+k_{2}^{2}} \frac{\partial \psi_{i}}{\partial x_{3}},(i=1,2), \quad \frac{\partial \psi_{1}}{\partial x_{1}}+\frac{\partial \psi_{2}}{\partial x_{2}}=-\frac{2}{1+k_{2}^{2}} \frac{\partial \phi}{\partial x_{3}}, \text { at } x_{3}=h,
$$

where $\Delta_{2}=\partial_{1}^{2}+\partial_{2}^{2}$ and $\sqrt{-\Delta_{2}}$ is a pseudo differential operator, see [9].

\section{Illustrative Example}

In this section, the dynamic response of the coated elastic half space, which is loaded by a tangential force, is evaluated within the framework of the asymptotic formulation derived in the previous section. Consider a tangential point load acting along the $x_{1}$ axis given by

$$
\mathbf{P}=\left(M \delta\left(x_{1}\right) \delta\left(x_{2}\right) \delta(t), 0,0\right) .
$$

From the decomposition introduced in (5), $\mathbf{P}$ may be written as

$$
\left(M \delta\left(x_{1}\right) \delta\left(x_{2}\right) \delta(t), 0,0\right)=\left(\frac{\partial P_{0}}{\partial x_{1}}+\frac{\partial P_{1}}{\partial x_{2}}, \frac{\partial P_{0}}{\partial x_{2}}-\frac{\partial P_{1}}{\partial x_{1}}, 0\right),
$$

resulting in

$$
\Delta_{2} P_{0}=M \delta^{\prime}\left(x_{1}\right) \delta\left(x_{2}\right) \delta(t),
$$


where $\Delta_{2}=\partial_{1}^{2}+\partial_{2}^{2}$ is two dimensional Laplacian. On using the well-known fundamental solution for two dimensional Laplace operator, [27,

$$
E\left(x_{1}, x_{2}\right)=\frac{1}{4 \pi} \ln \left(x_{1}^{2}+x_{2}^{2}\right),
$$

$P_{0}$ may be expressed as the convolution of eq. $(26)$ with the fundamental solution (27), namely

$$
P_{0}\left(x_{1}, x_{2}, t\right)=E\left(x_{1}, x_{2}\right) * M \delta^{\prime}\left(x_{1}\right) \delta\left(x_{2}\right) \delta(t)=\frac{M}{2 \pi} \frac{x_{1}}{x_{1}^{2}+x_{2}^{2}} \delta(t) .
$$

Thus, the hyperbolic equations on the surface $x_{3}=h$ are written as

$$
\begin{aligned}
& \Delta_{2} \psi_{1}-\frac{1}{c_{R}^{2}} \frac{\partial^{2} \psi_{1}}{\partial t^{2}}-b h \sqrt{-\Delta_{2}} \Delta_{2} \psi_{1}=M_{0} \frac{x_{2}^{2}-x_{1}^{2}}{\left(x_{1}^{2}+x_{2}^{2}\right)^{2}} \delta(t), \\
& \Delta_{2} \psi_{2}-\frac{1}{c_{R}^{2}} \frac{\partial^{2} \psi_{2}}{\partial t^{2}}-b h \sqrt{-\Delta_{2}} \Delta_{2} \psi_{2}=-2 M_{0} \frac{x_{1} x_{2}}{\left(x_{1}^{2}+x_{2}^{2}\right)^{2}} \delta(t),
\end{aligned}
$$

where

$$
M_{0}=\frac{M\left(1+k_{2}^{2}\right)}{4 \pi \mu B} .
$$

Introducing scaled variables

$$
\eta_{1}=\frac{x_{1}}{b h}, \quad \eta_{2}=\frac{x_{2}}{b h}, \quad \tau=\frac{c_{R}}{b h} t,
$$

the surface equations $(29)$ and 30 take the forms

$$
\begin{aligned}
& \frac{\partial^{2} \psi_{1}}{\partial \eta_{1}^{2}}+\frac{\partial^{2} \psi_{1}}{\partial \eta_{2}^{2}}-\frac{\partial^{2} \psi_{1}}{\partial \tau^{2}}-\sqrt{-\left(\frac{\partial^{2}}{\partial \eta_{1}^{2}}+\frac{\partial^{2}}{\partial \eta_{2}}\right)}\left(\frac{\partial^{2} \psi_{1}}{\partial \eta_{1}^{2}}+\frac{\partial^{2} \psi_{1}}{\partial \eta_{2}^{2}}\right)=\frac{M_{0} c_{R}}{b h} \frac{\eta_{2}^{2}-\eta_{1}^{2}}{\left(\eta_{1}^{2}+\eta_{2}^{2}\right)^{2}} \delta(\tau), \\
& \frac{\partial^{2} \psi_{2}}{\partial \eta_{1}^{2}}+\frac{\partial^{2} \psi_{2}}{\partial \eta_{2}^{2}}-\frac{\partial^{2} \psi_{2}}{\partial \tau^{2}}-\sqrt{-\left(\frac{\partial^{2}}{\partial \eta_{1}^{2}}+\frac{\partial^{2}}{\partial \eta_{2}}\right)}\left(\frac{\partial^{2} \psi_{2}}{\partial \eta_{1}^{2}}+\frac{\partial^{2} \psi_{2}}{\partial \eta_{2}^{2}}\right)=\frac{-2 M_{0} c_{R}}{b h} \frac{\eta_{1} \eta_{2}}{\left(\eta_{1}^{2}+\eta_{2}^{2}\right)^{2}} \delta(\tau) .
\end{aligned}
$$

Let us first consider the surface equation of shear potential $\psi_{1}$. Applying a double Fourier and a Laplace transform to eq. (33) result in

$$
\psi_{1}^{F F L}=-\frac{M_{0} c_{R}}{b h} \frac{\xi_{1}^{2}}{\left(\xi_{1}^{2}+\xi_{2}^{2}\right)\left(s^{2}+\left(\xi_{1}^{2}+\xi_{2}^{2}\right)\left(1-\sqrt{\xi_{1}^{2}+\xi_{2}^{2}}\right)\right)}
$$


where $\left(\xi_{1}, \xi_{2}\right)$ and $s$ are the Fourier and Laplace transform parameters, respectively. Taking the inverse double Fourier and Laplace transforms gives

$$
\begin{aligned}
\psi_{1}\left(\eta_{1}, \eta_{2}, \tau\right)=-\frac{M_{0} c_{R}}{4 \pi^{2} b h}\left\{\int_{\rho>1} \frac{\xi_{1}^{2} \mathrm{e}^{-\rho \sqrt{\rho-1} \tau}}{2 \rho^{3} \sqrt{\rho-1}} \mathrm{e}^{i \boldsymbol{\rho} \cdot \mathbf{r}} d \boldsymbol{\rho}\right. \\
\left.+\int_{\rho<1} \frac{\xi_{1}^{2} \sin (\rho \sqrt{1-\rho} \tau)}{\rho^{3} \sqrt{1-\rho}} \mathrm{e}^{i \boldsymbol{\rho}, \cdot \mathbf{r}} d \boldsymbol{\rho}\right\},
\end{aligned}
$$

where $\mathbf{r}=\left(\eta_{1}, \eta_{2}\right)=(r \cos \theta, r \sin \theta)$ and $\boldsymbol{\rho}=\left(\xi_{1}, \xi_{2}\right)=(\rho \cos \omega, \rho \sin \omega)$ with $|\mathbf{r}|=r$ and $|\boldsymbol{\rho}|=\rho$. The above integral may then be written as

$$
\begin{aligned}
\psi_{1}(r, \theta, \tau)=-\frac{M_{0} c_{R}}{4 \pi^{2} b h}\left\{\int_{1}^{\infty} \frac{\mathrm{e}^{-\rho \sqrt{\rho-1} \tau}}{2 \sqrt{\rho-1}} \int_{0}^{2 \pi} \cos ^{2} \omega \mathrm{e}^{i r \rho \cos (\omega-\theta)} d \omega d \rho\right. \\
\left.+\int_{0}^{1} \frac{\sin (\rho \sqrt{1-\rho} \tau)}{\sqrt{1-\rho}} \int_{0}^{2 \pi} \cos ^{2} \omega \mathrm{e}^{i r \rho \cos (\omega-\theta)} d \omega d \rho\right\} .
\end{aligned}
$$

Using the trigonometric relation for $\cos ^{2} \omega$ the first integral in the above equation can be written as

$$
\int_{0}^{2 \pi} \cos ^{2} \omega \mathrm{e}^{i r \rho \cos (\omega-\theta)} d \omega d \rho=\frac{1}{4} \int_{0}^{2 \pi}\left(\mathrm{e}^{2 i \omega}+\mathrm{e}^{-2 i \omega}+2\right) \mathrm{e}^{i r \rho \cos (\omega-\theta)} d \omega d \rho .
$$

Changing the variable $\omega-\theta=\gamma$, the first integral on the right hand side of (38) takes the form

$$
\int_{0}^{2 \pi} \mathrm{e}^{2 i \omega} \mathrm{e}^{i r \rho \cos (\omega-\theta)} d \omega=-\mathrm{e}^{2 i \theta} \int_{\theta_{0}}^{2 \pi+\theta_{0}} \mathrm{e}^{i(2 \gamma-r \rho \sin \gamma)} d \gamma=-2 \pi \mathrm{e}^{2 i \theta} J_{2}(r \rho),
$$

where $\theta_{0}=-\theta-\pi / 2$ and $J_{2}(r \rho)$ is Bessel function of the first kind defined as

$$
J_{n}(x)=\frac{1}{2 \pi} \int_{-\pi}^{\pi} \mathrm{e}^{i(x \sin \gamma-n \gamma)} d \gamma
$$

Similarly, the second and third integrals on the right hand side of 38 are written, respectively, as

$$
\int_{0}^{2 \pi} \mathrm{e}^{-i 2 \omega} \mathrm{e}^{i r \rho \cos (\omega-\theta)} d \omega=-2 \pi \mathrm{e}^{-2 i \theta} J_{-2}(r \rho)
$$


and

$$
2 \int_{0}^{2 \pi} \mathrm{e}^{i r \rho \cos (\omega-\theta)} d \omega=4 \pi J_{0}(r \rho) .
$$

The shear potential $\psi_{1}$, thus, can be expressed in terms of Bessel functions of the first kind as

$$
\begin{aligned}
\psi_{1}(r, \theta, \tau)=\frac{M_{0} c_{R}}{4 \pi b h}\left\{\int_{0}^{1} \frac{\sin (\rho \sqrt{1-\rho} \tau)}{\sqrt{1-\rho}}\left(\cos 2 \theta J_{2}(r \rho)-J_{0}(r \rho)\right) d \rho\right. \\
\left.+\int_{1}^{\infty} \frac{\mathrm{e}^{-\rho \sqrt{\rho-1} \tau}}{2 \sqrt{\rho-1}}\left(\cos 2 \theta J_{2}(r \rho)-J_{0}(r \rho)\right) d \rho\right\} .
\end{aligned}
$$

Repeating an almost identical procedure, the shear potential $\psi_{2}$ may be put in the form

$$
\begin{aligned}
\psi_{2}(r, \theta, \tau)=\frac{M_{0} c_{R}}{4 \pi b h} \sin 2 \theta\left\{\int_{0}^{1} \frac{\sin (\rho \sqrt{1-\rho} \tau)}{\sqrt{1-\rho}} J_{2}(r \rho) d \rho\right. \\
\left.+\int_{1}^{\infty} \frac{\mathrm{e}^{-\rho \sqrt{\rho-1} \tau}}{2 \sqrt{\rho-1}} J_{2}(r \rho) d \rho\right\} .
\end{aligned}
$$

It is known from eq. (14) that the normal displacement at the surface $x_{3}=h$ may be given in terms the wave potentials by

$$
\left.u_{3}\right|_{x_{3}=h}=\frac{\partial \phi}{\partial x_{3}}+\frac{\partial \psi_{2}}{\partial x_{1}}+\frac{\partial \psi_{1}}{\partial x_{2}}
$$

which can be expressed in terms of the new variables as

$$
\left.u_{3}\right|_{x_{3}=h}=\frac{1-k_{2}^{2}}{2 b h}\left(\cos \theta \frac{\partial \psi_{1}}{\partial r}-\frac{\sin \theta}{r} \frac{\partial \psi_{1}}{\partial \theta}+\sin \theta \frac{\partial \psi_{2}}{\partial r}+\frac{\cos \theta}{r} \frac{\partial \psi_{2}}{\partial \theta}\right) .
$$

On using the relation between the potentials (24), it is possible to write the scaled normal displacement from the related derivative of the integral expressions of $\psi_{1}$ and $\psi_{2}$ as

$$
U_{3}(r, \theta, \tau)=\cos \theta\left\{\int_{0}^{1} \frac{\sin (\rho \sqrt{1-\rho} \tau)}{\sqrt{1-\rho}} \rho J_{1}(r \rho) d \rho+\int_{1}^{\infty} \frac{\mathrm{e}^{-\rho \sqrt{\rho-1}} \tau}{2 \sqrt{\rho-1}} \rho J_{1}(r \rho) d \rho\right\},
$$

where

$$
U_{3}=\frac{4 \pi b^{2} h^{2}}{M c_{R}\left(1-k_{2}^{2}\right)} u_{3}
$$

As it is formidable to calculate the integrals in equation (45) analytically we employ numerical integration schemes to illustrate the surface displacement. 


\section{Numerical Results}

In this section numerical illustrations of the scaled longitudinal displacement $U_{3}$ defined in (45) are presented. Fig. 3 shows the variation of the vertical displacement $U_{3}$ on the variable $r$ depending on $\theta$ at $\tau=1$. As might be expected, the amplitude of the normal displacement $U_{3}$ decreases away from the surface load. Another important point is that the dispersive effect of the coating causes smoothing of the discontinuities, arising in the uncoated half-space problem, see 13. It should also be emphasized that the normal displacement becomes zero at $\theta=\pi / 2,3 \pi / 2$ because of the definition of $U_{3}$, see (45). Since we concerned with the tangential load applied on the surface, a load applied perpendicular to the surface results in nonzero displacement. The variation of the displacement $U_{3}$ on the angle $\theta$ for several values of the polar distance $r$ at $\tau=0.01$ is depicted in Fig. 4. Similar to the previous case, the magnitude of displacement decreases for the larger values of $r$. It is also observed that the displacement becomes zero at $\theta=\pi / 2$ and $\theta=3 \pi / 2$ because the applied load, then, becomes perpendicular.

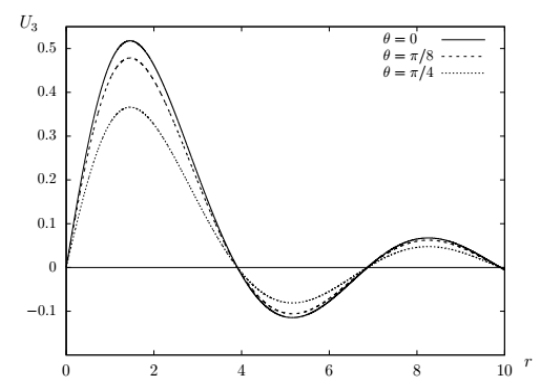

FiguRE 3 . The scaled vertical displacement $U_{3}$ versus $r$

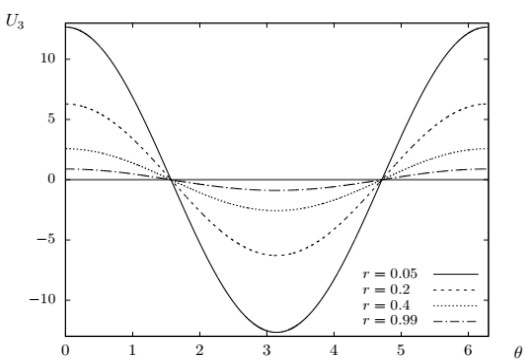

Figure 4 . The scaled vertical displacement $U_{3}$ versus $\theta$ 


\section{Conclusions}

In this paper, a 3D problem for a coated elastic half-space loaded by a tangential force along the surface is investigated. A long wave model for the coated half space derived in [9] and an asymptotic model for the in-plane surface wave of elastic half-space derived in [13] have been extended to the case of an in-plane loading for a three-dimensional elastic half-space coated by a thin layer. The problem is, then, formulated by two elliptic equations in the shear potentials $\psi_{1}$ and $\psi_{2}$ over the interior and a two-dimensional hyperbolic equation singularly perturbed by a pseudo-differential operator given along the interface $x_{3}=h$, see (24). The longitudinal and shear potentials are also related at $x_{3}=h$. It can be seen from the established model that the rotational part of the tangential load does not have any effect on the boundary equation clearly seen in (23). An integral solution of the normal displacement is expressed for the illustrative examples of point load acting on one of the in-plane axis. This solution shows that there is no displacement for a load applied perpendicular to the the surface of the half-space. It can be also observed that the presence of a coating results in smoothing the singularities arising in the corresponding problem of an uncoated half space, see [13.

The proposed approach may be generalized to more complicated structure including the effects of pre-stress, anisotropy, layered structures and viscosity, see [28-30]. The obtained asymptotic formulation may also be applied to in-plane moving load problems, see e.g. 20] and 21].

\section{REFERENCES}

[1] Rayleigh, J. W. S., On waves propagated along the plane surface of an elastic solid, Proc. Lond. Math. Soc. 17, (1885), 4-11.

[2] Friedlander, F. G., On the total reflection of plane waves, Quart. J. Mech. Appl. Math, 1, (1948), 376-384.

[3] Chadwick, P., Surface and interfacial waves of arbitrary form in isotropic elastic media, $J$. Elast, 6, (1976), 73-80.

[4] Kiselev, A. P. and Parker, D. F., Omni-directional Rayleigh, Stoneley and Schölte waves with general time dependence, Proc. R. Soc. Lond. A, 466(2120), (2010), 2241-2258.

[5] Knowles, J. K., A note on elastic surface waves, J. Geophys. Res., 71, (1966), 5480-5481.

[6] Kaplunov, J. D. and Kossovich, L. Y., Asymptotic model of Rayleigh waves in the far-field zone in an elastic half-plane, Dokl. Phys., 49, (2004), 234-236.

[7] Kaplunov, J. D., Kossovich, L., and Zakharov, A., An explicit asymptotic model for the Bleustein-Gulyaev wave, C. R. Mecanique, 332, (2004), 487-492.

[8] Kaplunov J., Zakharov A. and Prikazchikov D. A., Explicit models for elastic and piezoelastic surface waves, IMA J. Appl. Math., 71, (2006), 768-782.

[9] Dai, H. H., Kaplunov J. and Prikazchikov D.A., A long-wave model for the surface elastic wave in a coated half-space, Proc. R. Soc. A., 466, (2010), 3097-3116.

[10] Nobili, A., and Prikazchikov D.A., Explicit formulation for the Rayleigh wave field induced by surface stresses in an orthorhombic half-plane, European Journal of Mechanics-A/Solids, 70, (2018), 86-94. 
[11] Parker, D. F., The Stroh formalism for elastic surface waves of general profile, Proceedings of the Royal Society A: Mathematical, Physical and Engineering Sciences, 469(2160), (2013), 20130301.

[12] Kaplunov, J., Prikazchikov D., and Sultanova, L., Rayleigh-type waves on a coated elastic half-space with a clamped surface, Philosophical Transactions of the Royal Society A, $377(2156)$, (2019), 20190111.

[13] Ege, N., Erbaş, B., and Prikazchikov, D. A., On the 3D Rayleigh wave field on an elastic halfspace subject to tangential surface loads, ZAMM Journal of Applied Mathematics and Mechanics/Zeitschrift für Angewandte Mathematik und Mechanik, 95(12), (2015), 1558-1565.

[14] Kaplunov, J., and Prikazchikov, D.A., Asymptotic theory for Rayleigh and Rayleigh-type waves, In Advances in applied mechanics (Vol. 50, pp. 1-106). Elsevier, 2017.

[15] Erbaş, B., Kaplunov, J., Nolde, E., and Palsü, M., Composite wave models for elastic plates, Proceedings of the Royal Society A: Mathematical, Physical and Engineering Sciences, 474(2214), (2018), 20180103.

[16] Cole, J., Huth, J., Stresses produced in a half plane by moving loads, J. Appl. Mech., 25, (1958), 43-436.

[17] Freund, L.B., Wave motion in an elastic solid due to a nonuniformly moving line load, Quart. Appl. Math., 30, (1972), 27-281.

[18] Agostinacchio, M., Ciampa, D., Diomedi, M. and Olita, S., Parametrical analysis of the railways dynamic response at high speed moving loads, J. Mod. Transp., 21(3), (2013), 169181.

[19] Cao, Y., Xia, H. and Li, Z., A semi-analytical/FEM model for predicting ground vibrations induced by high- speed train through continuous girder bridge, J. Mech. Sci. Technol., 26(8), (2012), 2485-2496.

[20] Kaplunov, J. D., Prikazchikov, D. A., Erbaş, B., and Şahin, O., On a 3D moving load problem for an elastic half space, Wave Motion, 50(8), (2013), 1229-1238.

[21] Erbaş, B., Kaplunov, J., Prikazchikov, D. A., and Şahin, O., The near-resonant regimes of a moving load in a three-dimensional problem for a coated elastic half-space, Mathematics and Mechanics of Solids, 22(1), (2017), 89-100.

[22] Erbaş, B. and Şahin, O., On the causality of the Rayleigh wave, Journal of mechanics of materials and structures 11(4), (2016), 449-461.

[23] Ege, N., Sahin, O., and Erbas, B., Response of a 3D elastic half-space to a distributed moving load, Hacettepe Journal of Mathematics and Statistics, 46(5), (2017), 817-828.

[24] Şahin, O., and Ege, N., Surface displacement field of a coated elastic half-space under the Influence of a moving distributional load, Anadolu Üniversitesi Bilim Ve Teknoloji Dergisi-B Teorik Bilimler, 5(1), (2017), 77-90.

[25] Achenbach, J.D., Wave propagation in elastic solids, North-Holland, Amsterdam, 1973.

[26] Georgiadis, H. G. and Lykotrafitis, G., A method based on the Radon transform for threedimensional elastodynamic problems of moving loads, J. Elast., 65, (2001), 87-129.

[27] Polyanin, A. D., and Nazaikinskii, V. E., Handbook of linear partial differential equations for engineers and scientists. Chapman and hall/crc., 2015.

[28] Prikazchikov, D. A., Rayleigh waves of arbitrary profile in anisotropic media, Mechanics Research Communications, 50, (2013), 83-86.

[29] Shuvalov, A. L., and Every, A. G., On the long-wave onset of dispersion of the surface-wave velocity in coated solids, Wave Motion, 45(6), (2008), 857-863.

[30] Romeo, M., Rayleigh waves on a viscoelastic solid half-space, J. Acoust. Soc. Am., 110, (2001), 59-67. 
Current address: Onur Şahin: Giresun University, Faculty of Arts and Sciences, Department of Mathematics, Giresun, Turkey.

E-mail address: onur.sahin@giresun.edu.tr

ORCID Address: https://orcid.org/0000-0001-5389-9855 